\title{
Eficácia do extrato concentrado contendo Saccharum officinarum L. Poaceae, Azadirachta indica A. Juss. Meliaceae, e Eucaliptus spp Myrtaceae, sobre Pediculus capitis De Geer, (Anoplura: Pediculidae)
}

\author{
Rafael A. N. Ramos, Danillo S. Pimentel, Carlos A. N. Ramos, Maria A. G. Faustino, \\ Leucio C. Alves*
}

\begin{abstract}
Departamento de Medicina Veterinária, Universidade Federal Rural de Pernambuco, Av. Dom Manoel de Medeiros s/n, Dois Irmãos, 52171-900 Recife-PE, Brasil
\end{abstract}

\begin{abstract}
RESUMO: O objetivo deste trabalho foi avaliar a eficácia do extrato concentrado contendo Saccharum officinarum L. Poaceae, Azadirachta indica A. Juss. Meliaceae, e Eucaliptus spp Myrtaceae sobre Pediculus capitis De Geer Pediculidae. Foram coletados mil e trinta e cinco piolhos da cabeça de crianças infestadas por Pediculus capitis de creches da Região Metropolitana do Recife, Estado de Pernambuco, Brasil. Para realização do teste utilizou-se três grupos com trezentos e quarenta e cinco piolhos cada referentes aos produtos, extrato concentrado (EC), inseticida piretróide (PI) e controle (C). Os piolhos foram imersos em solução dos produtos por três minutos e em seguida secos. A mortalidade foi monitorada em diferentes momentos, por um período de vinte e quatro horas. O extrato concentrado matou todos os piolhos antes de doze horas e uma média de $60,28 \%$ (208/345) de mortalidade do EC ocorreu entre três e seis horas, a maior parte da mortalidade do PI foi observada $24 \mathrm{~h}$ após o tratamento. Os resultados mostraram que o extrato contendo Saccharum officinarum, Azadirachta indica, e Eucaliptus spp constitui-se como uma potente ferramenta no controle do Pediculus capitis.
\end{abstract}

Unitermos: medicina alternativa, pediculicida, piolho.

\begin{abstract}
Effectiveness of concentrate extract containing Saccharum Officinarum L. Poaceae, Azadirachta Indica A. Juss. Meliaceae, And Eucaliptus Spp Myrtaceae Against Pediculus Capitis De Geer, (Anoplura: Pediculidae)". The aim of this work was to evaluate the effectiveness of concentrate extract containing Saccharum officinarum L. Poaceae, Azadirachta indica A. Juss. Meliaceae, and Eucaliptus spp Myrtaceae against Pediculus capitis De Geer Pediculidae. A thousand and thirty five head lice were collected from children with Pediculus capitis infestation from some day care centers at Metropolitan Region of Recife, Pernambuco State, Brazil. The tests were performed in three groups with three hundred forty five lice each one according to product, concentrate extract (CE), pyrethroid insecticide (PI) and control (C). The immersing head lice in the diluted products for three minutes, washing off products and dry the insects were used. The mortality of lice was monitored at different points in time, for a period of twenty four hours. Concentrate extract killed all head lice after twelve hours and an average of $60.28 \%(208 / 345)$ of lice mortality of the CE occurred between three and six hours, while the mortality of PI was observed $24 \mathrm{~h}$ after treatment. The results showed the extract containing Saccharum officinarum, Azadirachta indica, and Eucaliptus spp could be a potent tool in the control of Pediculus capitis.
\end{abstract}

Keywords: alternative medicine, pediculicides, head lice.

\section{INTRODUÇÃO}

A infestação por Pediculus capitis De Geer Pediculidae no homem tem sido relatada desde os tempos mais remotos, sendo considerado um sério problema de saúde pública (Barbosa \& Pinto, 2003).

O parasitismo por esta espécie de piolho vem sendo descrito em várias regiões do mundo, acometendo principalmente crianças, notadamente com idade escolar (Kokturk et al., 2003; Morsy et al., 2001; Sim et al., 2003).

Segundo Barbosa \& Pinto (2003) vários medicamentos são disponíveis no mercado para a pediculose, dentre eles destacam-se os produtos a base de 
organofosforados e piretróides. Nenhuma dessas drogas é eficazmente segura, pois podem apresentar grandes efeitos colaterais em pacientes portadores de problemas respiratórios.

Por outro lado, relatos de resistência ao diclorodifenil-tricloroetano (DDT) (Maunder, 1991), assim como a permetrina, têm sido descritos em várias partes do mundo (Coz et al., 1993; Mumcuoglu et al., 1995; Pollack et al., 1999; Picollo et al., 2000).

Em vista desse problema se busca cada vez mais o desenvolvimento de novas moléculas para controle desses ectoparasitos. Sendo assim, o objetivo do presente trabalho foi avaliar a eficácia do extrato concentrado contendo Saccharum officinarum L. Poaceae, Azadirachta indica A. Juss. Meliaceae e Eucaliptus spp Myrtaceae sobre Pediculus capitis em condições de laboratório.

\section{MATERIAL E MÉTODOS}

Os espécimes foram coletados diretamente do couro cabeludo de crianças de ambos os sexos e idades variadas provenientes de diferentes creches da Cidade do Recife. Os piolhos foram conduzidos ao Laboratório de Doenças Parasitárias dos Animais Domésticos da UFRPE.

Para realização do teste utilizou-se mil e trinta e cinco espécimes adultos divididos em três grupos contendo trezentos e quarenta e cinco piolhos referentes ao extrato concentrado contendo Saccharum officinarum, Azadirachta indica e Eucaliptus spp (EC), o piretróide inseticida $(\mathrm{PI})$ e ao controle $(\mathrm{C})$ respectivamente. Para o grupo controle utilizou-se água destilada.

Os piolhos foram imersos em solução dos produtos a serem testados, durante três minutos, após os quais se retirou o excesso de líquido, sendo os mesmos colocados em placas de Petri revestidas com papel filtro para monitoramento durante $24 \mathrm{~h}$ a temperatura ambiente. Para tanto foram realizadas cinco observações a partir de $1,3,6,12$ e 24 h após o início do teste. qui-quadrado.

Foi realizada a análise estatística pelo teste $\chi^{2}$ -

\section{RESULTADOS E DISCUSSÃO}

Os resultados obtidos na avaliação estão descrito na Tabela 1 .

De acordo com os dados apresentados na Tabela 1, observou-se que no grupo do extrato contendo Saccharum officinarum L. Poaceae, Azadirachta indica A. Juss. Meliaceae e Eucaliptus spp Myrtaceae (CE) todos os espécimes morreram em até doze horas após o início do teste. Observou-se também que 60,28\% (208\345) dos piolhos deste grupo morreram nos períodos compreendidos entre três e seis horas, mostrando uma ação mais rápida do extrato quando comparado ao piretróide inseticida (PI).

$\mathrm{O}$ controle $(\mathrm{C})$ mostrou-se viável durante todo o teste, mesmo sem alimentação, sendo a mortalidade observada neste grupo decorrentes da biologia do inseto.

A ação de extratos e óleos essenciais vegetais contra ectoparasitos vem sendo observada em todo o mundo (Yang et al., 2005; Audino et al., 2007). Algumas substâncias encontradas nestes extratos como terpenóides e alcalóides contribuem para sua atividade inseticida (Isman, 2001).

No presente estudo o fitoterápico in vitro mostrouse eficaz no controle do Pediculus capitis, provavelmente pela ação inseticida do neem, tendo sido potencializado pelos outros componentes.

Os resultados do presente estudo indicam que o extrato concentrado contendo Saccharum officinarum, Azadirachta indica e Eucaliptus spp constitui-se como potente piolhicida no controle do Pediculs capitis.

Tabela 1. Mortalidade dos insetos após imersão nos produtos testados segundo o tempo de avaliação.

\begin{tabular}{lccccc}
\hline \multirow{2}{*}{ Tratamento } & \multicolumn{5}{c}{ Tempo (horas) } \\
\cline { 2 - 6 } & 1 & 3 & 6 & 12 & 24 \\
\hline Extrato concentrado (EC) & 93 & 106 & 102 & 44 & - \\
Piretróide inseticida (PI) & 53 & 48 & 62 & 92 & 90 \\
Controle & 31 & 42 & 41 & 42 & 189
\end{tabular}

\section{REFERÊNCIAS BIBLIOGRÁFICAS}

Audino PG, Vassena C, Zerba E, Picollo M 2007. Evectiveness of lotions based on essential oils from aromatic plants against permethrin resistant Pediculus humanus capitis. Arch Dermatol Res 392: 299-389.

Barbosa JV, Pinto ZT 2003. Pediculose no Brasil. Entomol Vect 10: $579-586$

Coz J, Combescot-Lang C, Verdier V 1993. Resistance du pou tete Pediculus capitis L. 1758 aux pyrethrinoides d-phenothrine et permethrine en France. $B \mathrm{Soc} F r$ Parasitol 2: 245-252.

Isman MB 2001. Pesticides based on plant essential oils for management of plant pests and diseases. Korea Forest Res Inst 19: 603-608.

Kokturk A, Baz K, Bugdayci R, Sasmaz T, Tursen U, Kaya TI, Ikizoglu G 2003. The prevalence of pediculosis capitis in schoolchildren in Mersin Turkey. Int J Dermatol 42: 694-698.

Maunder JW 1991. Strategic aspects of insecticide resistance in head lice. J Royal Soc of Health 125: 27-29.

Morsy TA, el-Ela RG, Mawla MY, Khalaf SA 2001. The prevalence of lice infesting students of primary, preparatory and secondary schools in Cairo, Egypt. $J$ Egypt Soc Parasitol 31: 43-50.

Mumcuoglu KY, Hemingway J, Miller J, loffe-Uspensky I, Klaus S, Ben-Ishai F, Galun R 1995. Permethrin resistance in the head louse Pediculus capitis from Israel. Med Vet 
Entomol 9: 427-432.

Picollo MI, Vassena C, Mougabure CG, Vernetti M, Zerba E 2000. Resistance to insecticides and effects of synergists on permethrin toxicity in Pediculus capitis (Phthiraptera, Pediculidae) from Buenos Aires. J Med Entomol 37: 721-725.

Pollack RJ, Kiszewski A, Armstrong P, Hahn C, Wolfe N, Rahman HA, Laserson K, Telford SR, Spielman A 1999. Differential permethrin susceptibility of head lice sampled in the United States and Borneu. Arch Pediatr Adolesc Med 153: 969-973.

Sim S, Lee IY, Lee KJ, Seo JH, Im KI, Shin MH Yong TS 2003. A survey on head lice infestation in Korea and the therapeutic efficacy of oral trimethoprim sulfamethoxazole adding to lindane shampoo. Korean J Parasitol 41: 57-61.

Yang YC, Lee HS, Lee SH, Clark JM, Ahn YJ 2005. Ovicidal and adulticidal activities of Cinnamomum zeylanicum bark essential oil compounds and related compounds against Pediculus humanus capitis (Anoplura: Pediculicidae). Int J Parasitol 35: 1595-1600. 